\title{
Análise epidemiológica do Acidente Vascular Cerebral no Brasil
}

\author{
Sara Regina Meira Almeida \\ Fisioterapeuta, Especialista em Fisioterapia Neurológica, Mestre e doutoranda em Ciências Médicas \\ pela Faculdade de Ciências Médicas da Unicamp, Campinas-SP, Brasil.
}

Em escala mundial, o acidente vascular cerebral (AVC) é a segunda principal causa de morte. É uma doença que ocorre predominantemente em adultos de meia-idade e idosos ${ }^{1,2}$. Nas últimas décadas, o Brasil vem mudando o seu perfil de morbimortalidade, com as doenças crônicas não transmissíveis liderando as principais causas de morte. Entre as mais importantes doenças crônicas está o AVC, que é uma das principais causas de internaçóes e mortalidade, causando na grande maioria dos pacientes, algum tipo de deficiência, seja parcial ou completa ${ }^{1}$.

No Brasil, foram registradas 160.621 internaçôes por doenças cerebrovasculares em 2009, segundo os dados de domínio público do Sistema Único de Saúde (DATASUS) ${ }^{3}$, do Ministério da Saúde. A taxa de mortalidade foi de 51,8 a cada grupo de 100.000 habitantes. O grupo acima de 80 anos representou quase 35\% dos 99.174 óbitos $^{3}$.

O estudo de Pinheiro e Vianna ${ }^{4}$ analisou essa temática, sobre a taxa de mortalidade numa região específica do Brasil. Os autores avaliaram o comportamento da taxa de mortalidade específica (TME) por AVC, na população do Distrito Federal, entre 1995 e 2005. De acordo com os resultados, houve uma redução da TME no sexo masculino entre 30 e 79 anos, com aumento da taxa nos indivíduos acima de 80 anos. Quanto ao sexo feminino, houve aumento da TME entre 30 e 49 anos, e na faixa etária acima dos 80 anos.

De Carvalho et al. (2011) também realizaram estudo epidemiológico de AVC no Brasil, mostrando uma maior prevalência do sexo feminino $(51,8 \%)$ no grupo de 2407 pacientes. Pinheiro e Vianna ${ }^{4}$ mostraram maior prevalência de óbito das mulheres quando comparadas a homens, em 2007.

Alguns aspectos como melhor condição socioeconômica, qualidade da prevenção primária e qualidade dos cuidados hospitalares podem ter auxiliado a diminuir a taxa de novos casos de AVC no Brasil. Além do melhor controle dos fatores de risco, como hipertensão arterial, diabetes, fibrilação atrial, tabagismo, sedentarismo e obesidade .

Como Pinheiro e Vianna discutem no artigo ${ }^{4}$, o sistema DATASUS não permite avaliar de forma quantitativa a influência dos fatores de risco na mortalidade, além de não disponibilizar informaçôes a respeito de sobrevida, após o ictus e o processo de reabilitação. Essas informações seriam importantes para melhorar e ampliar as iniciativas de saúde pública para a prevenção e o tratamento de AVC, seja na fase aguda ou crônica.

Embora após um AVC, normalmente, ocorra certo grau de retorno motor e funcional, muitos sobreviventes apresentam consequências crônicas que são, usualmente, complexas e heterogêneas, podendo resultar problemas em vários domínios da funcionalidade. Essa funcionalidade se refere à capacidade de realizar atividades do dia-a-dia, seja no aprendizado e aplicação de conhecimentos (atenção, pensamento, cálculos, resolução de problemas); na comunicação (linguagem falada, escrita); na mobilidade (manutenção da posição corporal, transferências, deambulaçáo); no autocuidado, vida doméstica, interaçáo interpessoal e social ${ }^{6}$. (Scheper et al, 2007).

Além das campanhas governamentais que estimulam a população a controlar melhor os fatores de risco da doença e da intervenção eficaz da equipe neurológica, na abordagem inicial de um paciente com AVC, seja isquêmico ou hemorrágico é importante também, a orientação e o acompanhamento pela equipe interdisciplinar. A fisioterapia, fonoaudiologia e terapia ocupacional irão atuar no processo de reabilitação, em longo prazo, possibilitando a reinserção do indivíduo à sociedade e a suas atividades diárias. 


\section{REFERÊNCIAS}

1.The WHO stepwise approach to stroke surveillance (endereco na internet). Overview and Manual (version2.0). Local: Noncommunicable Diseases and Mental Health. World Health Organization. (atualizado em: 01/2006 mes/ ano; acessado em 11/12. Disponivel em: http://www.who.int/ncd_surveillance/en/steps_stroke_manual_v1.2.pdf

2.Pontes-Neto OM, Silva GS, Feitosa MR, de Figueiredo NL, Fiorot JA, Rocha TN, et al. Stroke awareness in Brazil. Stroke 2008;39:292-6.

http://dx.doi.org/10.1161/STROKEAHA.107.493908

3.Ministério da Saúde/SE/Datasus (endereço na internet).Local: Sistema de Informações Hospitalares do SUS - SIH/SUS IBGE: base demográfica. (atualizado em: 12/2010; acessado em: 11/2012) Disponivel em: http://www2. datasus.gov.br/DATASUS/index.php?area $=02$
4.Pinheiro HA, Vianna LG. Taxa de Mortalidade Específica por Doenças Cerebrovasculares no Distrito Federal entre 1995 e 2005. Rev Neurocienc 2012;20(4):488-493.

http://dx.doi.org/10.4181/RNC.2012.20.483.6p

5.de Carvalho JJF, Alves MB, Viana GAA, Machado CB, Santos BFC, Kanamura AH, et al. Stroke Epidemiology, Patterns of Management, and Outcomes in Fortaleza, Brazil - A Hospital-Based Multicenter Prospective Study. Stroke 2011;42:3341-6.

http://dx.doi.org/10.1161/STROKEAHA.111.626523

6.Scheper VP, Ketela M, Van DPI, Visser-Meily JM, Lindeman E. Comparing contents of functional outcome measures in stroke rehabilitation using the international classification of functioning, disability and health. Disabil Rehabil. 2007;29(3):221-30.

http://dx.doi.org/10.1080/09638280600756257 